9. H. Schöneborn, Über Linearformenmoduln unendlichen Ranges. I. Primäre, kompakte Linearformenmoduln, J. Reine Angew. Math. vol. 189 (1951) pp. 168-185.

10. N. Vilenkin, Direct decompositions of topological groups. I, II, Mat. Sbornik vol. 19 (1946) pp. 85-154, 311-340. Amer. Math. Soc. Translation no. 23.

11. - On the theory of weakly separable groups, Mat. Sbornik vol. 22 (1948) pp. 135-177.

12. D. Zelinsky, Linearly compact modules and rings, Amer. J. Math. vol. 75 (1953) pp. 79-90.

University of Chicago

\title{
A PROOF OF HESSENBERG'S THEOREM
}

\section{ARNO CRONHEIM}

About fifty years ago Hessenberg [1] discovered that in a projective plane the Pappus property implies the Desargues property. In all the proofs of this beautiful theorem [2-5] only the so-called general case is treated so that some of the points and lines constructed in the course of the proof may actually become indeterminate. In the following lines we offer a proof of this theorem which takes care of all possible cases. We show first how to reduce the discussion to two cases only. The first of these cases may be treated by Hessenberg's argument, but in the second one a different proof is needed.

We consider now a projective plane. Whenever the points $X, Y$, and $Z$ are collinear, then we write $(X, Y, Z)$; and the fact that they are not collinear we indicate by writing $\operatorname{non}(X, Y, Z)$. In order to prove the Desarguesian closure property for our projective plane it suffices to consider seven distinct points $A_{i}, B_{i}$, and $S$ such that the three lines $A_{i}+B_{i}$ are distinct and meet in $S$ and neither $\left(A_{1}, A_{2}, A_{3}\right)$ nor $\left(B_{1}, B_{2}, B_{3}\right)$. The following two properties of such a configuration are independent of the Pappus property.

(1) non $\left(A_{i}, B_{i}, B_{k}\right)$ and $\operatorname{non}\left(A_{i}, A_{k}, B_{k}\right)$ for $i \neq k$.

Proof. This is obvious since $S+A_{i}+B_{i} \neq S+A_{k}+B_{k}$ for $i \neq k$.

(2) If there does not exist a permutation $(i, j, k)$ of the numbers $(1,2,3)$ such that $\operatorname{non}\left(A_{i}, B_{j}, B_{k}\right)$ and $\operatorname{non}\left(B_{k}, A_{i}, A_{j}\right)$ simultaneously, then either $\left(A_{x}, B_{y}, B_{z}\right)$ for all permutations $(x, y, z)$ or $\left(B_{x}, A_{y}, A_{z}\right)$ for all permutations $(x, y, z)$.

Proof. Let us assume without loss of generality non $\left(B_{1}, A_{2}, A_{3}\right)$. It follows from the hypothesis of (2) that $\left(A_{2}, B_{1}, B_{3}\right)$ and $\left(A_{3}, B_{1}, B_{2}\right)$. $\left(B_{3}, A_{1}, A_{2}\right)$, together with $\left(A_{2}, B_{1}, B_{3}\right)$, would contradict

Received by the editors May 18, 1952. 
non $\left(A_{1}, A_{2}, B_{1}\right)$. We have therefore non $\left(B_{3}, A_{1}, A_{2}\right)$ and, by hypothesis of (2), $\left(A_{1}, B_{2}, B_{3}\right)$.

Now we denote by $P_{i}$ the well determined point $\left(A_{j}+A_{k}\right)\left(B_{j}+B_{k}\right)$ where $(i, j, k)$ is again a permutation of $(1,2,3)$. We want to prove that these points $P_{i}$ are collinear, provided the Pappus property is valid in the projective plane under consideration.

We distinguish two cases.

Case 1 . There exists a permutation $(i, j, k)$ such that non $\left(A_{i}, B_{j}, B_{k}\right)$ and $\operatorname{non}\left(B_{k}, A_{i}, A_{j}\right)$.

In this case Hessenberg's classical argument may be used, which we sketch now for the convenience of the reader. Let us assume that non $\left(A_{1}, B_{2}, B_{3}\right)$ and non $\left(B_{3}, A_{2}, A_{1}\right)$. We define $Q=\left(A_{1}+A_{2}\right)\left(B_{3}+B_{2}\right)$. Since we have non $\left(A_{1}, B_{2}, B_{3}\right)$ and non $\left(A_{i}, B_{2}, B_{3}\right)(i=2,3), Q \neq A_{i}$; similarly $Q \neq B_{i} . S+A_{1} \neq S+A_{2}$ implies $Q \neq S$. We consider now the two triples $\left(A_{3}, B_{3}, S\right)$ and $\left(Q, A_{2}, A_{1}\right)$. These six points are different and, since non $\left(A_{1}, A_{2}, A_{3}\right)$, lie on different lines. We get therefore a well defined Pappus configuration with $\left(X, E, P_{1}\right)$, where $X$ $=\left(A_{2}+B_{2}+S\right)\left(A_{1}+B_{3}\right)$ and $E=\left(A_{1}+A_{3}\right)(S+Q)$. In taking the two triples $\left(B_{1}, A_{1}, S\right)$ and $\left(Q, B_{2}, B_{3}\right)$ we get similarly $\left(X, F, P_{3}\right)$, where $F=\left(B_{1}+B_{3}\right)(S+Q)$. Next we consider the two triples $(F, Q, E)$ and $\left(A_{1}, X, B_{3}\right)$. We show that none of the points $F, Q$, and $E$ lies on $A_{1}+B_{3}$. $\left(Q, A_{1}, B_{3}\right)$, together with $\left(Q, B_{2}, B_{3}\right)$, would contradict non $\left(A_{1}, B_{2}, B_{3}\right) . E=A_{1}$, together with $(S, Q, E)$ and $\left(Q, A_{1}, A_{2}\right)$, would contradict $S+A_{1} \neq S+A_{2}$. Therefore $E \neq A_{1}$. Now $\left(E, A_{1}, B_{3}\right)$, together with $\left(E, A_{1}, A_{3}\right)$, would contradict non $\left(A_{1}, A_{3}, B_{3}\right)$. Quite similarly we get non $\left(F, A_{1}, B_{3}\right)$. Furthermore $A_{1}, X$, and $B_{3}$ are distinct, since $X=A_{1}$ would imply $\left(A_{2}, B_{2}, A_{1}\right)$ and $X=B_{3}$ would imply $\left(A_{2}, B_{2}, B_{3}\right)$, both in contradiction to (1). We have therefore a well defined Pappus configuration and get $\left(P_{1}, P_{2}, P_{3}\right)$.

Case 2. There does not exist a permutation $(i, j, k)$ such that $\operatorname{non}\left(A_{i}, B_{j}, B_{k}\right)$ and $\operatorname{non}\left(B_{k}, A_{i}, A_{j}\right)$.

It follows from (2) that either $\left(A_{x}, B_{y}, B_{z}\right)$ or $\left(B_{x}, A_{y}, A_{z}\right)$ for all permutations $(x, y, z)$. We assume without loss of generality the validity of $\left(A_{1}, B_{2}, B_{3}\right),\left(A_{2}, B_{1}, B_{3}\right)$, and $\left(A_{3}, B_{1}, B_{2}\right)$.

We remark in the beginning that $P_{i} \neq A_{k}$ for all $i, k$, since neither $\left(A_{i}, A_{j}, A_{k}\right)$ nor $\left(A_{j}, B_{j}, B_{k}\right)$; similarly $P_{i} \neq B_{k}$. We define $Q_{1}$ $=\left(B_{3}+P_{3}\right)\left(S+A_{1}\right)$ and $Q_{2}=\left(B_{3}+P_{3}\right)\left(S+A_{2}\right)$. Since $P_{3}$ is on $B_{1}+B_{2}$, but is different from $B_{1}, B_{2}$, and $A_{3}$, we remark that $B_{3}+P_{3}$ is different from the three lines $B_{3}+B_{1}+A_{2}, B_{3}+B_{2}+A_{1}$, and $B_{3}+A_{3}+S$. The points $P_{3}, Q_{i}, A_{i}, B_{i}$, and $S$ are therefore distinct. Since non $\left(A_{1}, A_{2}, A_{3}\right)$ the two triples $\left(S, A_{3}, B_{3}\right)$ and $\left(P_{3}, A_{2}, A_{1}\right)$ lie on different lines. The Pappus property implies $\left(P_{2}, Q_{1}, B_{2}\right)$. Consider- 
ing $\left(S, A_{3}, B_{3}\right)$ and $\left(P_{3}, A_{1}, A_{2}\right)$ we get $\left(P_{1}, Q_{2}, B_{1}\right)$. Next we consider $\left(Q_{1}, A_{1}, B_{1}\right)$ and $\left(A_{2}, Q_{2}, B_{2}\right)$. Since $A_{1}+B_{1} \neq A_{2}+B_{2}, P_{1}=\left(B_{2}+B_{3}+A_{1}\right)$ $\left(B_{1}+Q_{2}\right), P_{2}=\left(B_{1}+B_{3}+A_{2}\right)\left(B_{2}+Q_{1}\right)$, and $P_{3}=\left(Q_{1}+Q_{2}\right)\left(A_{1}+A_{2}\right)$, the Pappus property finally implies $\left(P_{1}, P_{2}, P_{3}\right)$.

I am grateful to Professor R. Baer for helpful remarks.

\section{Bibliography}

1. G. Hessenberg, Beweis des Desarguesschen Satzes aus dem Pascalschen, Math. Ann. vol. 61 (1905) pp. 161-172.

2. - Grundlagen der Geometrie, Berlin, 1930.

3. K. Reidemeister, Grundlagen der Geometrie, Berlin, 1930.

4. G. de B. Robinson, The foundations of geometry, Toronto, 1940.

5. H. S. M. Coxeter, The real projective plane, New York, 1949.

UNIVERSITY OF ILLINOIS 